\title{
WHAT THEORISTS THINK THEY KNOW ABOUT AGB STARS
}

\author{
JOHN C. LATTANZIO \\ Department of Mathematics, Monash University \\ Clayton, VIC 3168, Australia
}

\begin{abstract}
In this paper I discuss our basic picture of the structure of AGB stars, and how their earlier evolution led them to this situation.
\end{abstract}

\section{Introduction}

The present paper is intended as an introduction to the structure of low and intermediate mass Asymptotic Giant Branch stars. It should provide a useful pointer into the literature for non-experts, and also serve to ensure that everyone understands the basic theory so that we can use this to debug the data!

\section{Discussion}

We discuss the evolution of a low mass star $\left(1 M_{\odot}\right)$ and an intermediate mass star $\left(5 M_{\odot}\right)$ in a purely qualitative sense. The evolution is followed from the ZAMS through to the AGB. The emphasis is on the internal structure, with particular reference to mixing and nucleosynthesis. After discussing core hydrogen exhaustion and the First Dredge-up, we briefly discuss semiconvection (which is seen in the low mass stars during core helium burning). Following core helium exhaustion the stars ascend the AGB. The more massive models will experience the Second Dredge-Up. Both models converge to a similar qualitative structure as they ascend the AGB and begin their thermally pulsing evolution.

After discussing the detailed structure and convection changes during a thermal pulse cycle, we discuss the position and extent of the ${ }^{13} \mathrm{C}$ pocket, which appears necessary for the production of $s$-process elements in these stars. This ${ }^{13} \mathrm{C}$ was initially believed to be burned when it was engulfed by the convective pocket at the next thermal pulse, but recent calculations indicate that the ${ }^{13} \mathrm{C}$ burns radiatively (Straniero et al. 1995) during the 
inter-pulse phase. We also discuss how thermal pulses can produce ${ }^{19} \mathrm{~F}$ (but we defer to Mowlavi et al. 1996 for details).

We then discuss the phenomenon of "hot bottom burning," where the convective envelope penetrates the top of the hydrogen burning shell. This process appears to explain the existence of super Li-rich stars (Boothroyd \& Sackmann 1992) as well as the lack of carbon stars at high luminosity (Wood, Bessell \& Fox 1983). The effect on other elements, such as CNO and ${ }^{26} \mathrm{Al}$, is also discussed. We then emphasise the importance of ion-probe analysis of meteoritic grains as a source of highly accurate quantitative information about stellar compositions (e.g. Zinner 1995).

\section{Further Reading}

Due to space limitations, this paper is not presented in this volume, but two very similar papers can be found in Frost \& Lattanzio (1996) and Lattanzio et al. (1996).

\section{References}

Boothroyd, A.I. \& Sackmann, I.-J. 1992, ApJ, 393, L21

Frost, C. A. \& Lattanzio, J. C. 1996, in Stellar Evolution: What Should Be Done?, Proceedings of the 32nd Liège Colloquium, ed. A. Noels et al., p. 307

Lattanzio, J. C., Frost, C. A., Cannon, R. C. \& Wood, P. R. 1996, Mem. Astron. Soc. Italia, 67,729

Mowlavi, N., Jorissen, A. \& Arnould, M. 1996, $A \& A, 311,803$

Straniero, O., Gallino, R., Busso, M., Chieffi, A., Raiteri, C. M., Limongi, M. \& Salaris, M. 1995, ApJ, 440, L85

Wood, P. R., Bessell, M.S. \& Fox, M. W. 1983, ApJ, 272, 99

Zinner, E.K. 1995, in Nuclei in the Cosmos III, ed. M. Busso, R. Gallino and C. M. Raiteri (AIP Press: New York), p. 567

\section{Discussion}

Wagenhuber: Do you use the mixing-length theory for your HBB calculations?

Lattanzio: The evolution calculations are carried out with the mixinglength theory and assume instantaneous mixing. For the time-dependent mixing required in the nucleosynthesis calculations we use a phenomenological method due to Cannon (1993, MNRAS, 263, 817). This requires a convective velocity and a local mixing length as input. At present we take these from the standard mixing-length theory. But the method is actually a combination of a "conveyor belt" model and a diffusion equation. Although it uses some inputs from the mixing-length theory, it is quite different in construction. 
Plez: From observations it seems we do not see the signature of the ${ }^{22} \mathrm{Ne}$ neutron source in any AGB stars. What does the theorist say about that?

Lattanzio: The models of low-mass stars do not produce temperatures high enough for this source (except for a very brief period near the peak of the pulse) so this is consistent with the models. Of course, the models do not at present produce the ${ }^{13} \mathrm{C}$ required either! But this is a problem with the details of convective boundaries, so we can be excused (at least a little).

Little-Marenin: Do your models give $s$-process enhancements (such as Tc) and mixing to the surface?

Lattanzio: At present we do not calculate any $s$-processing. We include a small iron-peak network so that we can estimate the neutron density, but we do not attempt to determine the details of the $s$-processing. This is at present impossible to do in a self-consistent manner, because it requires a small pocket of ${ }^{13} \mathrm{C}$, and how to obtain this is still a matter of some debate. Nevertheless, for reasonable assumptions about the size of this ${ }^{13} \mathrm{C}$ pocket, Gallino and collaborators can obtain very good agreement with observations of $s$-process elements (see Gallino et al. 1995, in the Proceedings of the "Nuclei in the Cosmos" meeting). The agreement is further improved by the recent discovery that the ${ }^{13} \mathrm{C}$ burns radiatively during the intershell phase rather than during ingestion into the convective pocket at the next pulse.

Whitelock: There are galactic stars with high $\mathrm{Li}$ abundances and masses not much more than one solar mass. Do we understand how these form?

Lattanzio: The standard picture requires $\mathrm{HBB}$ on the $\mathrm{AGB}$, and this requires a larger stellar mass. Boothroyd and Sackmann estimate that the Li-rich AGB stars have masses between about 4 and $6 M_{\odot}$, and our calculations agree with that. Nevertheless, Boothroyd (private communication) is able to produce Li enhancements in low-mass first giant branch stars by a process of deep mixing on the giant branch (also known as "cool bottom processing"), if the mixing is not too fast. There seems to be observational evidence for this effect on the first giant branch.

Some of the galactic carbon stars which are rich in ${ }^{7} \mathrm{Li}$ may also form from this mechanism: their mass is too small for HBB. But if cool processing occurs on the first giant branch, then it may also occur on the AGB. Of course, if it is rotationally induced then braking on the RGB may mean there is no mixing on the AGB. We just don't know. I believe (Boothroyd, private communication) that some observations (e.g. ${ }^{18} \mathrm{O}$ abundances) are indicative of mixing on the AGB, but are not conclusive. 